\title{
Nanomagnet-Based Detoxifying Machine: An Alternative/Complementary Approach in HIV therapy
}

Khaled Seidi' ${ }^{1}$, Ali Eatemadi ${ }^{1}$, Behzad Mansoori², Rana Jahanban-Esfahlan ${ }^{1,3 *}$ and Davoud Farajzadeh ${ }^{4,5 *}$

${ }^{1}$ Department of Medical Biotechnology, Faculty of Advanced Medical Sciences, Tabriz University of Medical Sciences, Tabriz, Iran

${ }^{2}$ Department of Immunology, Faculty of Medicine, Tabriz University of Medical Sciences, Tabriz, Iran

${ }^{3}$ Student Researches Committee, Tabriz University of Medical Sciences, Tabriz, Iran

${ }^{4}$ Department of Cellular and Molecular Biology, Faculty of Biological Science, Azarbaijan Shahid Madani University, Tabriz, Iran

${ }^{5}$ Department of Genetic, Biotechnology Research Center, Research \& Development Complex, Tabriz University of Medical Sciences, Tabriz, Iran

\begin{abstract}
Although the use of highly active antiretroviral therapy (HAART) hampers HIV-1 replication, it is not yet a cure. The main obstacle to HAART-mediated HIV eradication is the latent reservoir of virally infected cells, which is the source of fast viral rebound in patients that stopped therapy. Patients must therefore have lifelong HAART, which is expensive and in long term the many side effects undermine successful treatment. Attempts to eradicate latent reservoir have focused on reactivating latent proviruses. However, killing the infected cells after virus reactivation, which is pivotal for elimination of the reservoir, has not been addressed. In this paper, we proposed a novel complementary approach, which we have called the "Nanomagnet-Based Detoxifying Machine (NBDM)" and, is able to eliminate the virus load in the bloodstream of the patients. In this regard, our proposed nanomagnets (Fe3O4-PLGA-PEG-gp120 Ablaptamer) MIP) will be able to bind to any gp120 markers in the blood circulation. The nanomagnets will trap the virus particles in a dialysis column during the hemodialysis and under a strong electromagnetic field. Therefore, each nanomagnets will serve as a hook to capture millions of virus particles out of the patient's body. In the present research, we suggested the application of the Molecularly Imprinted Polymers (MIPs), because they could simply be synthesized through selfassembling approaches without any need for highly expensive Ab/aptamers. The synthesized MIPs have the potential to mimic the behavior of an antibody/aptamer raised against one of the main antigenic determinants of the virus, such as gp120. NBDM provides a simple but very beneficial system to detoxify the blood from both the viruses and nanoparticles. In conclusion, we think that it could be used as complementary therapy beside the HAART to improve the CD4+ count and disease prognosis without any concern for side effects. This is a simple, cost effectiveness, easy to handle, convenient for the patients, non-invasive, and effective approach that could be extended for a large spectrum of human diseases other than HIV.
\end{abstract}

Keywords: Molecularly Imprinted Polymers (MIPs); NanomagnetBased Detoxifying Machine (NBDM); HIV therapy

\section{Background}

AIDS or acquired immune deficiency syndrome is a pandemic disease that is caused by the human immunodeficiency virus 1 or 2 (HIV1 or 2). HIV-1 spreads through the sexual, prenatal and percutaneous routes. Hence, it is easily transmitted among individuals [1].

AIDS is a lethal multi-spectrum disease with a variety of manifestations. The disease is latent in the early stages, with a high probability of showing no symptoms for the first two or three years. After the latent stage, symptoms of the disease slowly become visible. These symptoms are divided into early symptoms (fever, sweating, malaise, lethargy, anorexia, nausea, myalgia, arthralgia, headache, sore throat, diarrhea, lymphadenopathy, and rash) and late symptoms (opportunistic infections, malignancy and neurological disorders) [2] .

According to the World Health Organization (WHO) report, the number of people worldwide who are living with HIV reached 35.3 million in 2012; 1.6 million deaths were attributed to AIDS in the same year [3]. Despite these telling statistics, there is still no guaranteed cure for this lethal disease. These statistics as well as the prevalence and spread of AIDS across the world, have led to the demand for increasing research in the treatment and cure for this deadly disease. In recent years, a wide variety of therapies has been tested. Unfortunately, none of these has resulted in an effective method of treatment. HIV therapy often relies on highly active antiretroviral therapy (HAART) [4]. With the advent of HAART, increased survival rates and changes in the epidemiological facts were noted. HAART, in combination with antiretroviral agents, is used to suppress virus replication. Hence, it causes a decrease in HIV diversity related to mutations in the HIV genome as well as HIV drug resistance [5]. The therapies-based HAART is a combination of antiretroviral agents, such as nucleotides reverse transcriptase inhibitors, nucleoside reverse transcriptase inhibitors, non-nucleoside reverse transcriptase inhibitors, protease inhibitors, and fusion inhibitors [6]. After treatment of patients living with HIV, a rapid decrease in the level of HIV was detected in the patients' blood; six months later, there were no signs of HIV-related disease and, the count of CD4 cells gradually increased as well. Several studies have shown that HAART can reduce the mortality of people living with HIV [7]. Further, adherence to HAART can be noticed in clear clinical

*Corresponding author: Davoud Farajzadeh, Department of Genetic Biotechnology Research Center, Research \& Development Complex, Tabriz University of Medical Sciences, Tabriz, Iran; Department of Cellular and Molecular Biology, Faculty of Biological Science, Azarbaijan Shahid Madani University, Tabriz, Iran, E-mail: farajzadeh@azaruniv.ac.ir

Rana Jahanban-Esfahlan, Department of Medical Biotechnology, Faculty of Advanced Medical Sciences, Tabriz University of Medical Sciences, Tabriz, Iran, E-mail: jahanbanr@tbzmed.ac.ir

Received March 11, 2014; Accepted April 24, 2014; Published May 11, 2014

Citation: Seidi K, Eatemadi A, Mansoori B, Jahanban-Esfahlan R, Farajzadeh D (2014) Nanomagnet-Based Detoxifying Machine: An Alternative/Complementary Approach in HIV therapy. J AIDS Clin Res 5: 304. doi:10.4172/2155-6113.1000304

Copyright: (c) 2014 Seidi K, et al. This is an open-access article distributed under the terms of the Creative Commons Attribution License, which permits unrestricted use, distribution, and reproduction in any medium, provided the original author and source are credited. 
results, reduction of mortality rate and the improvement of quality of life [8]. However, it has been reported that HAART can have many side effects, such as fatigue, nausea, vomit, diarrhea, and lipodystrophy, which in turn causes non-adherence to the treatment and, consequently result in increased level of blood viral count, decreased level of CD4 cell count, increased resistance of the HIV to the drugs, treatment failure, opportunistic infections, and loss of financial resources [9]. The use of monoclonal antibodies is another therapy. According to the morphological structure of HIV, the most important therapeutic target is gp120, which has led to monoclonal antibodies (mAbs), in turn, becoming increasingly popular therapeutic agents due to its relevant features. However, in reality, the problem is more extensive and goes beyond what it is thought to be. Although antibodies have been identified that neutralize over 90 percent of circulating HIV-1 isolates, they are not conclusively accepted as an effective treatment for HIV [10]. Administration of mAbs carries the risk of immune reactions [1114], infections [15-18], platelet and thrombotic disorders, autoimmune diseases, cancer, dermatitis and, cardiotoxicity [19-23].

Envelope glycoprotein 120 (or gp120) is a glycoprotein expressed on the outer layers of both infectious and non-infectious viruses, and it is present in the tissues and blood of patients living with HIV. In addition, gp120 plays a vital role in the entry of the virus into cells [24,25].

Several therapies have focused on the reactivation of the latent HIV-1 reservoir, which is the main barrier that hamper the efficacy of current therapies design to eradicate HIV-1 [26-28]. However, killing the infected cells after virus reactivation, which is essential for elimination of the reservoir, has not been addressed. Here we describe a simple and practical method that could efficiently eradicate HIV particles from the bloodstream of infected patients.

\section{Hypothesis}

The most important feature of the nanomagnets is how they are designed to capture gp120- bearing particles. To that end, three approaches could be used: antibodies, aptamers, or MIPs. In the present research, we suggest the application of the molecularly imprinted polymers (MIPs) as a new, emerging technology in designing our special nanomagents, which we have called the "Targeted nanomagnets" (T-nanomagnets). In this way, the MIP will serve as a template that can precisely mimic the actions of an antibody or aptamer against one of the major HIV markers (for example, gp120 receptors). The MIPs could be easily synthesized through self-assembling without any need for highly expensive Ab/aptamers [29-31]. Although antibodies have considered as the most successful affinity tools with diagnostic and therapeutic applications in both fundamental and applied research nowadays. Nonetheless, they do have several limitations, such as high production costs and low stability. MIPs are alternative affinity tools based on inorganic matrices (molecular imprinted polymers) that have received extensive attention. A major advantage of MIPs includes the efficient microbial production and in vitro selection procedures. Although MIPs are certainly not as widely used as antibodies, the number of their applications has progressively increased [29,31-34].

Thanks to the tiny size of nanoparticles, which allows them to enter any small capillaries within tissues, and also their enhanced circulation time, it is expected that the application of MIPs in the structure of magnetic nanoparticles (nanomagnets) will eliminate virus load in the blood. These nanomagnets will circulate in the patients' blood and gather intelligently any particles carrying the HIV marker gp120. The Nanomagnet-Based Detoxifying Machine (NBDM) requires the simple and easy accessible materials to start, i.e, Targeted-nanomagnets $\left(\mathrm{Fe}_{3} \mathrm{O}_{4}\right.$ PLGA-PEG-MIP) and magnet column. This protocol consists of three steps as following: synthesis of targeted nanomagnets $\left(\mathrm{Fe}_{3} \mathrm{O}_{4}\right.$-PLGAPEG-MIP), Injection of targeted nanomagnets to the patients' blood and finally the dialysis of the patients' blood. The proposed structure for T-nanomagnets is shown in Figure 1.

\section{General Specifications}

The efficacy of the approach can be affected by various factors listed below, which need to be optimized.

1) The size of the iron oxide that will determine its super paramagnetic property.

2) The preferred shape of the iron oxide.

3) The preferred magnetization of the particles: the thickness of the PLGA-PEG coating and the loading efficiency of iron oxide are parameters that will influence the magnetization.

4) The preferred surface density of PEG and the anti-gp120.

5) The intensity of electromagnetic field should be optimized in order to avoid the possible rheological changes in blood.

It is also an important advantage for NBDM that does not require expensive membranes while the inner surface of the magnet column serves as an attachment surface for the T-nanomagnets. However, to
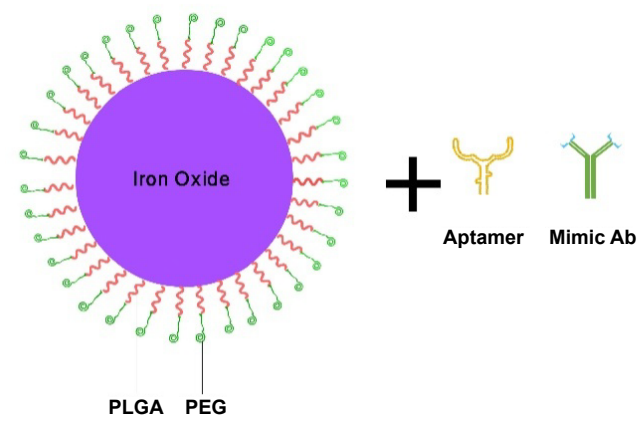

Magnet nanoparticle

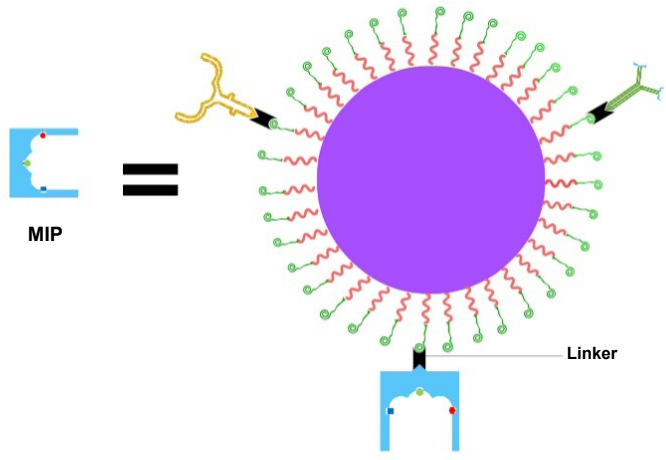

Targeted Magnet nanoparticle

Figure 1: Illustrative structure of proposed T-nanomagnet. 
increase the surface area, it is possible to divide the inside part into small compartments. The column may have an internal volume to hold 300 to $500 \mathrm{ml}$ of the blood. The volume of involved connection silicone-tubes may not to be exceeded $200 \mathrm{ml}$. Hence, the total volume of blood being processed at a given point of time would be less than 700 $\mathrm{ml}$. The flow rate may be between 100 and $300 \mathrm{ml} \mathrm{min}^{-1}$; however, the settings would be adjusted according to the treatment conditions.

\section{Evaluation of the Hypothesis}

\section{Synthesis of targeted nanoparticles}

Recently, nanoparticles have gained increasing popularity. One group of nanoparticles is magnet nanoparticles. It is shown that some metals, such as cobalt, nickel, cadmium, zinc, and silver can be toxic biologically and hence may not to be safe for the clinical applications while other metals, such as titanium and iron oxide are less toxic to the cells $[35,36]$. Thus, we recommend the use of iron oxides. In addition, to minimize the toxicity, the coated-nanoparticles have been suggested. For this purpose, there are many coating materials, such as PLGA-PEG, PCL-PEG and PLA-PEG. In this paper, the PLGA-PEG coated by iron oxide nanoparticles is considered.

In the next step, the potential therapeutic targets should be selected. The best potential targets for therapy can be a surface marker. The two main potential markers that have attracted interest as potential therapeutic targets are gp120 and gp41. Gp120 is coded by the HIV env gene and is attached to viral envelop with a transmembrane glycoprotein, gp41. HIV1 envelope spikes include three gp120 attached to three gp41 in a trimer of heterodimers $[37,38]$. Gp120 has a potential for attachment to many receptors, such as a CD4 receptor, DC-SIGN,

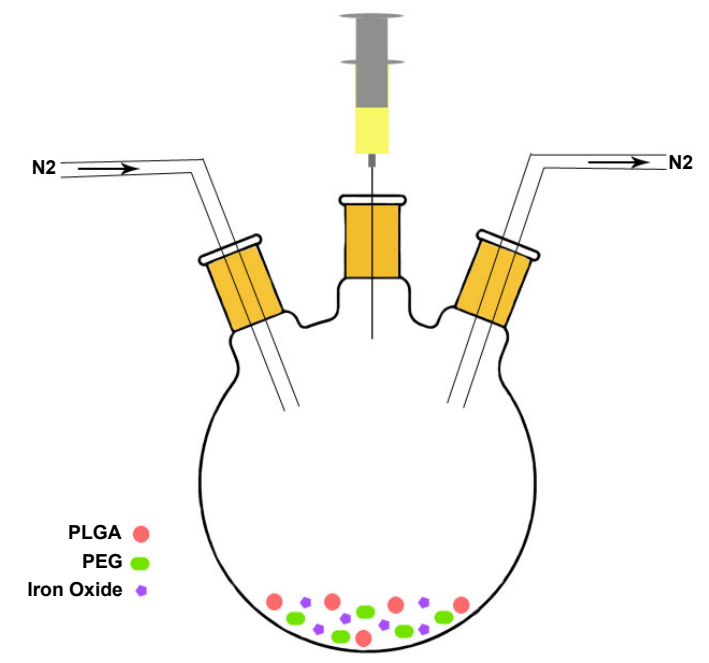

Figure 2: Synthesis of PLGA-PEG coated by Iron oxide nanoparticles. The initial structure of Nanomagnets is prepared using an improved chemical coprecipitation method ${ }^{29}$. According to this method, $\mathrm{FeCl}_{2} \cdot 4 \mathrm{H}_{2} \mathrm{O}$ and $\mathrm{FeCl}_{3} \cdot 6 \mathrm{H}_{2} \mathrm{O}$ are dissolved in deionized water and stirred under nitrogen atmosphere at $80^{\circ} \mathrm{C}$ for 1 hour. Then, $\mathrm{NH}_{3} \cdot \mathrm{H}_{2} \mathrm{O}$ is rapidly injected. After cooling in the room temperature, the precipitated particles are washed and separated by magnetic decantation, and are dried under vacuum at $70^{\circ} \mathrm{C}$. In next step, PLGA-PEG is prepared according to the ring opening polymerization method $[42,43]$. Briefly, Lactide, glycolide and PEG are mixed in three necked bottle under nitrogen atmosphere at $1400^{\circ} \mathrm{C}$. Then stannous octoate $(0.05 \% \mathrm{w} / \mathrm{w})$ are added. The solution is stirred under nitrogen atmosphere at $1400^{\circ} \mathrm{C}$ for 8 hours. After cooling at room temperature, the mixture is dissolved in dichloromethane. Finally, the solution is precipitated in the cold chloroform.

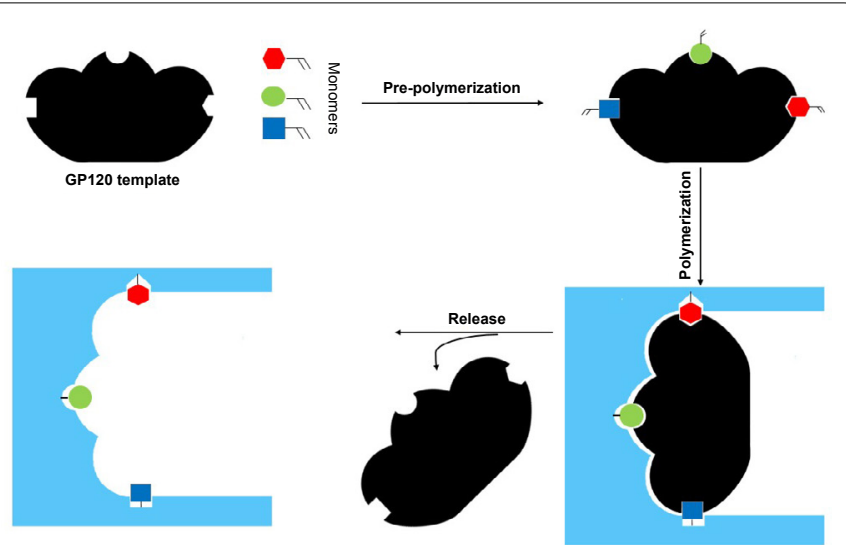

Figure 3: Scheme of molecular imprinting against gp120. Gp120 receptors are chosen as the target and imprint molecule for this hypothesis. First step is to consider the gp120 monomers and template (template is a constant, short peptide sequence illustrative of an available fragment of a larger protein, holes on the template are representative of the active sites on the gp120 template). Afterwards, all elements of the MIPs are combined and allowed to be self-assembled to form the cross-linked polymer with the template (prepolymerization). After polymerization, monomers and the surrounding matrix are cleaved from the template molecules. The resulting targeted MIPs will be able selectively to bind to gp120.

heparan sulfate proteoglycan, CXCR4, and CCR5 [38-44], making it a suitable marker to trap the HIV virus by T-nanomagnets. The method for synthesis of T-nanomagnets is depicted in Figure 2 and 3. Detailed instructions for the synthesis of MIP are available in literature $[45,46]$.

\section{Administration of synthesized T-Nanomagnets to patients and blood dialysis}

An optimized dose of T-nanomagnets will be injected to patients intravenously and, within 24 hours, a patient's blood will be applied to the Nanomagnet-Based Detoxifying Machine (NBDM). As mentioned earlier, NBDM is a device that uses a magnetic field to trap magnet nanoparticles in a magnet column. The magnet column takes its name from the magnets that cover its outer surface to provide the maximum capacity for binding of T-nanomagnets under a strong, constant electromagnetic field. This system implies the presence of an input source injected into the infected blood, into the magnet column, and an output source to deliver the virus-nanomagnet depleted blood back to a patient's body (Figure 4).

Dialysis could be repeated in appropriate intervals to eradicate as many viruses as possible. The virus count could initially be used as an indicator for efficacy of the dialysis; later, the number of CD4+ cells could predict the proper time for adjuvant therapy like HAART.

\section{Consequences of the hypothesis - clinical significance}

The simplicity and safety of NBDM suggests its use as a routine therapeutic intervention for physicians to clear bloodstreams, not only for removing the lethal HIV viruses from patients' blood, but it might cover a wide range of human implications, such as various human infectious diseases, different subsets of solid and hematologic cancers, etc. All that is needed is a specific marker to be used in MIP design. For example, in the case of prostate cancer, you need a template that could mimic the properties of an Ab/aptamer against the prostatic specific antigen (PSA), or in case of breast cancer, you can use an MID for a Her-2Neu antigen. In the case of solid tumors, we don't expect to 


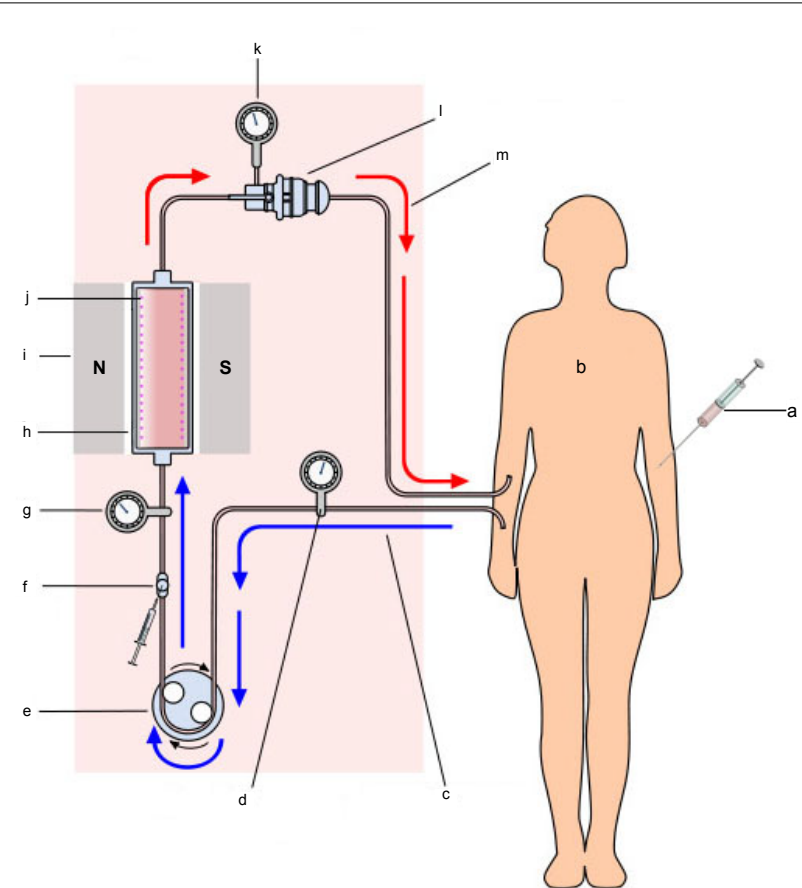

Figure 4: Schematic diagram of proposed "NBDM" to eliminate of HIV and gp120 from the patients' blood treated by T-nanaomagnets. Main features include: (a) Syringe containing T-nanomagnets (b) patient (c) arterial blood (d) arterial pressure monitor (e ) blood pump (f) heparin pump (g) dialyzer inflow pressure monitor $(\mathrm{h})$ dialyzer (i) electromagnetic field (j) magnetic nanoparticles $(k)$ venous pressure monitor, (I) air trap and air detector, and $(\mathrm{m})$ T-nanomagnet and gp120 cleaned-blood injects to patient.

cure but any decrease in the load of circulated tumor specific antigens would improve the efficacy of subsequent therapeutic interventions. NBDM especially could be used in advanced stages of disease when the high load of pathogen in patient's system hinder effective therapeutic interventions

\section{Conclusion and Prospective}

HIV is an infectious disease that feeds and survives on human immune system cells. By far, there is no efficient and safe therapy to control and cure the devastating effects of this virus on the human immune system. The key to successfully controlling HIV lethal symptoms initially depends on the load of virus and the number of CD4+ cells. While the latency is the main barrier to HIV eradication, some researchers tried ways to reactivate the latent reservoir. However a reliable method to clear these activated viruses needs to be accessed. A magneted-based dialysis system could meet this demand, not only in case of HIV, but could also be amenable for other infectious lifethreading diseases to improve the prognosis of the disease. Nontoxic nanomagnets described in this paper, could circulate freely and capture viruses residing in any possible part of the body, and finally exposure to a magnetic field will remove both nanomagnets and the attached viruses. Subsequently, blood starvation will make the virus harmless as soon as it gets out of bloodstream by means of sophisticated T-nanomagnets. Due to all advantages mentioned in the application of NBDM, we believe that it could be used as regular complementary therapy for efficient HIV targeted therapeutics.

\section{References}

1. Pickrell J (2006) Timeline: HIV and AIDS. New Scientist.
2. Michelle G, Workman C (2009) Clinical manifestations and the natural history of HIV. HIV Management in Australasia, 125-132.

3. http://www.who.int/hiv/pub/me/unaids_global_report/en/index.html .

4. Castro A (2005) Adherence to antiretroviral therapy: merging the clinical and social course of AIDS. PLoS Med 2: e338.

5. Gulick RM, Mellors JW, Havlir D, Eron JJ, Gonzalez C, et al. (1997) Treatment with indinavir, zidovudine, and lamivudine in adults with human immunodeficiency virus infection and prior antiretroviral therapy. N Engl J Med 337: 734-739.

6. Levi GC, Vitória MA (2002) Fighting against AIDS: the Brazilian experience. AIDS 16: 2373-2383.

7. Reis AC, Santos EMd, Cruz MMd (2007) A mortalidade por aids no Brasil: um estudo exploratório de sua evolução temporal. Epidemiologia e Serviços de Saúde 16: 195-205.

8. Chiou PY, Kuo BI, Lee MB, Chen YM, Chuang P, et al. (2006) A programme of symptom management for improving quality of life and drug adherence in AIDS/ HIV patients. J Adv Nurs 55: 169-179.

9. Ruiz-Perez I, Olry de Labry-Lima A, Prada-Pardal JL, Rodriguez-Bano J Causse-Prados M, et al. (2006) Impact of demographic and psychosocial factors on adherence to antiretroviral treatment. Enferm Infecc Microbiol Clin 24: 373-378.

10. Wu X, Yang ZY, Li Y, Hogerkorp CM, Schief WR, et al. (2010) Rational design of envelope identifies broadly neutralizing human monoclonal antibodies to HIV-1. Science 329: 856-861

11. Presta LG (2006) Engineering of therapeutic antibodies to minimize immunogenicity and optimize function. Adv Drug Deliv Rev 58: 640-656.

12. Klastersky $J$ (2006) Adverse effects of the humanized antibodies used as cancer therapeutics. Curr Opin Oncol 18: 316-320.

13. Kang SP, Saif MW (2007) Infusion-related and hypersensitivity reactions of monoclonal antibodies used to treat colorectal cancer--identification, prevention, and management. J Support Oncol 5: 451-457.

14. Ransohoff RM (2007) Natalizumab for multiple sclerosis. N Engl J Med 356 2622-2629.

15. Keane $\mathrm{J}$ (2005) TNF-blocking agents and tuberculosis: new drugs illuminate an old topic. Rheumatology (Oxford) 44: 714-720.

16. Bongartz T, Sutton AJ, Sweeting MJ, Buchan I, Matteson EL, et al. (2006) AntiTNF antibody therapy in rheumatoid arthritis and the risk of serious infections and malignancies: systematic review and meta-analysis of rare harmful effects in randomized controlled trials. JAMA 295: 2275-2285.

17. Schneeweiss S, Setoguchi S, Weinblatt ME, Katz JN, Avorn J, et al. (2007) Antitumor necrosis factor alpha therapy and the risk of serious bacterial infections in elderly patients with rheumatoid arthritis. Arthritis Rheum 56: 1754-1764.

18. Major EO (2009) Reemergence of PML in natalizumab-treated patients--new cases, same concerns. N Engl J Med 361: 1041-1043.

19. Aster RH, Bougie DW (2007) Drug-induced immune thrombocytopenia. N Eng J Med 357: 580-587.

20. Ramos-Casals M, Brito-Zeron P, Munoz S, Soria N, Galiana D, et al. (2007) Autoimmune diseases induced by TNF-targeted therapies: analysis of 233 cases. Medicine (Baltimore) 86: 242-251.

21. Dixon W, Silman A (2006) Is there an association between anti-TNF monoclona antibody therapy in rheumatoid arthritis and risk of malignancy and serious infection? Commentary on the meta-analysis by Bongartz et al. Arthritis Res Ther 8: 111.

22. Agero AL, Dusza SW, Benvenuto-Andrade C, Busam KJ, Myskowski P, et al (2006) Dermatologic side effects associated with the epidermal growth factor receptor inhibitors. J Am Acad Dermatol 55: 657-670.

23. Cardinale D, Colombo A, Torrisi R, Sandri MT, Civelli M, et al. (2010) Trastuzumab-induced cardiotoxicity: clinical and prognostic implications of troponin I evaluation. J Clin Oncol 28: 3910-3916.

24. Chougnet C, Gessani S (2006) Role of gp120 in dendritic cell dysfunction in HIV infection. J Leukoc Biol 80: 994-1000.

25. Jones MV, Bell JE, Nath A (2000) Immunolocalization of HIV envelope gp120 in HIV encephalitis with dementia. AIDS 14: 2709-2713. 
Citation: Seidi K, Eatemadi A, Mansoori B, Jahanban-Esfahlan R, Farajzadeh D (2014) Nanomagnet-Based Detoxifying Machine: An Alternative/ Complementary Approach in HIV therapy. J AIDS Clin Res 5: 304. doi:10.4172/2155-6113.1000304

Page 5 of 5

26. Marini A, Harper JM, Romerio F (2008) An in vitro system to model the establishment and reactivation of HIV-1 latency. J Immunol 181: 7713-7720.

27. Bosque A, Planelles V (2009) Induction of HIV-1 latency and reactivation in primary memory CD4+ T cells. Blood 113: 58-65.

28. Brooks DG, Hamer DH, Arlen PA, Gao L, Bristol G, et al. (2003) Molecular characterization, reactivation, and depletion of latent HIV. Immunity 19: 413423.

29. Cheong WJ, Yang SH, Ali F (2013) Molecular imprinted polymers for separation science: a review of reviews. J Sep Sci 36: 609-628.

30. Li Y, Yang HH, You QH, Zhuang ZX, Wang XR (2006) Protein recognition via surface molecularly imprinted polymer nanowires. Anal Chem 78: 317-320.

31. Bergmann NM, Peppas NA (2008) Molecularly imprinted polymers with specific recognition for macromolecules and proteins. Progress in Polymer Science 33: 271-288.

32. Bossi A, Bonini F, Turner AP, Piletsky SA (2007) Molecularly imprinted polymers for the recognition of proteins: the state of the art. Biosens Bioelectron 22: 1131-1137

33. Bures P, Huang Y, Oral E, Peppas NA (2001) Surface modifications and molecular imprinting of polymers in medical and pharmaceutical applications. J Control Release 72: 25-33

34. Hilt JZ, Byrne ME (2004) Configurational biomimesis in drug delivery: molecular imprinting of biologically significant molecules. Adv Drug Deliv Rev 56: 15991620.

35. Berry CC (2005) Possible exploitation of magnetic nanoparticle-cell interaction for biomedical applications. Journal of Materials Chemistry 15: 543-547.

36. Hofmann-Amtenbrink M, Hofmann H, Montet X (2010) Superparamagnetic nanoparticles - a tool for early diagnostics. Swiss Med Wkly 140: w13081.

37. Center RJ, Leapman RD, Lebowitz J, Arthur LO, Earl PL, et al. (2002)
Oligomeric structure of the human immunodeficiency virus type 1 envelope protein on the virion surface. J Virol 76: 7863-7867.

38. Zhu P, Chertova E, Bess J, Lifson JD, Arthur LO, et al. (2003) Electron tomography analysis of envelope glycoprotein trimers on HIV and simian immunodeficiency virus virions. Proc Natl Acad Sci U S A 100: 15812-15817.

39. Curtis BM, Scharnowske S, Watson AJ (1992) Sequence and expression of a membrane-associated C-type lectin that exhibits CD4-independent binding of human immunodeficiency virus envelope glycoprotein gp120. Proc Natl Acad Sci U S A 89: 8356-8360.

40. Dalgleish AG, Beverley PC, Clapham PR, Crawford DH, Greaves MF, et al. (1984) The CD4 (T4) antigen is an essential component of the receptor for the AIDS retrovirus. Nature 312: 763-767.

41. de Witte L, Bobardt M, Chatterji U, Degeest G, David G, et al. (2007) Syndecan-3 is a dendritic cell-specific attachment receptor for HIV-1. Proc Natl Acad Sci U S A 104: 19464-19469.

42. Lin G, Baribaud F, Romano J, Doms RW, Hoxie JA (2003) Identification of gp120 binding sites on CXCR4 by using CD4-independent human immunodeficiency virus type 2 Env proteins. J Virol 77: 931-942.

43. Napier KB, Wang ZX, Peiper SC, Trent JO (2007) CCR5 interactions with the variable 3 loop of gp120. J Mol Model 13: 29-41.

44. Pancera M, Majeed S, Ban YE, Chen L, Huang CC, et al. (2010) Structure of HIV-1 gp120 with gp41-interactive region reveals layered envelope architecture and basis of conformational mobility. Proc Natl Acad Sci U S A 107: 1166-1171.

45. Akbarzadeh A, Mikaeili H, Zarghami N, Mohammad R, Barkhordari A, et al. (2012) Preparation and in vitro evaluation of doxorubicin-loaded $\mathrm{Fe}_{3} \mathrm{O}_{4}$ magnetic nanoparticles modified with biocompatible copolymers. Int J Nanomedicine 7 : $511-526$

46. Ito R, Machida Y, Sannan T, Nagai T (1990) Magnetic granules: a nove system for specific drug delivery to esophageal mucosa in oral administration. International Journal of Pharmaceutics 61: 109-117. 